\title{
Lesiones por accidentes de tránsito en una institución de salud en el municipio de Pereira entre los años 2014-2017
}

\author{
Injuries from traffic accidents in a health institution in the municipality of Pereira from 2014 to 2017
}

Isabel Trujillo-Trejos ${ }^{1}$ orcid.org/0000-0003-3810-8802

Eliana Soley Gutiérrez-Calderón'1 orcid.org/0000-0001-8820-4216

Erika Giraldo-Castañeda' ${ }^{1}$ orcid.org/0000-0002-7202-4656

Gabriel Antonio Grisales-Giraldo1,2 orcid.org/0000-0002-7212-6660

Andrés A. Agudelo-Suárez ${ }^{1,3^{*}}$ orcid.org/0000-0002-8079-807X

1 Fundación Universitaria del Área Andina - Seccional Pereira. Pereira, Colombia

2 Grupo de Investigación Hospital Universitario San Jorge. Pereira, Colombia

3 Facultad de Odontología. Universidad de Antioquia. Medellín, Colombia tránsito en una institución de salud en el municipio de Pereira entre los años 2014-2017. Univ. Salud. 2019;21(1):8-18. D0I: http://dx.doi.org/10.22267/rus.192101.135

\section{Resumen}

Introducción: Los accidentes de tránsito se han convertido en un importante problema de salud pública debido a su alta morbimortalidad. La tendencia en lesiones y muerte por accidentes de tránsito se encuentra en niveles alarmantes en países como Colombia. Objetivo: Caracterizar epidemiológicamente las lesiones por accidentes de tránsito reportados en personas atendidas en una institución de salud en el municipio de Pereira entre 2014 y 2017. Materiales y métodos: Estudio descriptivo y transversal basado en fuentes secundarias según características sociodemográficas, epidemiológicas y clínicas de los accidentes de tránsito atendidos en una entidad de salud del municipio de Pereira, durante el período comprendido entre los años 2014 a 2017. Resultados: Se analizaron 460 casos en el periodo estudiado (64,1\%) en hombres. El motociclista representó el 44,3\% de las víctimas. El 64,3\% sufrieron contusiones, seguido del 20,9\% de fracturas, el 53\% de las lesiones se presentaron en miembros superiores e inferiores. Conclusiones: Existió un incremento significativo de las lesiones ocasionadas por accidentes de tránsito, producidas por motociclistas y peatones, asociadas a la impericia del conductor y la distracción del peatón, el cual desencadenó en contusiones y fracturas y aumento de la carga de la enfermedad.

Palabras clave: Accidentes de tránsito; heridas y lesiones; peatones; salud pública. (Fuente: DeCS, Bireme).

\begin{abstract}
Introduction: Traffic accidents have become an important public health problem due to their high morbimortality. The trend in injury and death from road traffic accidents is at alarming levels in countries like Colombia. Objective: To characterize epidemiologically the injuries by traffic accidents reported in people who were served in a health institution in the municipality of Pereira from 2014 to 2017. Materials and methods: A descriptive and transversal study was made based on secondary sources according to sociodemographic, epidemiological and clinical characteristics of traffic accidents attended by a health entity in the municipality of Pereira from 2014 to 2017. Results: 460 cases were analyzed in the studied period, $64.1 \%$ in males. The motorcyclist represented $44.3 \%$ of the victims. $64.3 \%$ suffered bruises, followed by $20.9 \%$ of fractures. $53 \%$ of the lesions were presented in upper and lower limbs. Conclusions: There was a significant increase in injuries caused by traffic accidents caused by motorcyclists
\end{abstract}


and pedestrians, associated with the driver's incompetence and the distraction of the pedestrian, which triggered in contusions, fractures and increased burden of disease.

Key words: Accidents, traffic; wounds and injuries; pedestrians; public health. (Source: DeCS, Bireme).

\section{Introducción}

Los accidentes de tránsito (AT) actualmente representan un problema de salud pública que va en aumento; según el Informe sobre la Situación Mundial de la Seguridad Vial 2015 de la Organización Mundial de Salud (OMS), las lesiones y cualquier tipo de alteración, producto de los accidentes de tránsito son la séptima causa de mortalidad en el mundo y la primera entre los jóvenes de 15 a 29 años. El $90 \%$ de las muertes derivadas de los AT, ocurren en países en vía de desarrollo y subdesarrollo, quienes aportan el $54 \%$ de los vehículos mundiales. Cerca de 1,25 millones de personas mueren producto de AT cada año, además, se calcula que existen entre 20 a 50 millones de traumatismos no mortales anuales, aumentando la carga económica y social de la enfermedad(1).

Un accidente de tránsito es una colisión o un incidente en el que se encuentre un vehículo en movimiento en una vía pública o privada; puede incluirse a la colisión entre dos vehículos, vehículo y peatón, animales, obstáculos y sólo un vehículo(2).

Las carreteras de todo el mundo presentan múltiples obstáculos, desde su infraestructura hasta sus mecanismos de protección, aumentando el riesgo de mortalidad. Dicha mortalidad depende en gran ocasión de lugar o país de residencia, el cual se ve asociado a su progreso vial, y vías de acceso. En América, la prevalencia de defunciones en motocicleta en relación al total de defunciones por accidentes de tránsito se incrementó de un 15\% al $20 \%$ entre los años 2010 y 2013. En las regiones de Asia y Pacífico occidental los motociclistas representan una tercera parte de los AT(3,4). Los peatones y los ciclistas también están entre los grupos más vulnerables y representan respectivamente, un $22 \%$ y un $4 \%$ de las defunciones mundiales, asociado al consumo de alcohol(1,5,6).
En Colombia, el Instituto de Medicina Legal y Ciencias Forenses, reportó para el año 2017, 48.869 casos de accidentes de tránsito, con un saldo de 6.754 muertos y 40.115 lesionados. En Risaralda la tasa departamental fue de 13,4 muertes en accidente de tránsito, por cada 100.000 habitantes/año, dato similar al nacional que fue de 13,7 en el año 2017(7). En este mismo año el informe sobre violencia y accidentalidad en el departamento, reportó que los accidentes de tránsito cobraron la vida de 129 personas, el mayor número de accidentes con desenlace fatal ocurrieron en Pereira, Dosquebradas, Santa Rosa de Cabal y La Virginia; siendo los hombres quienes tienen mayor riesgo de sufrir lesiones en accidente de tránsito, encontrándose una razón hombre:mujer de 2:1. La edad de los lesionados en ambos sexos estuvo comprendida entre los 20 y 24 años. Para este mismo año, Pereira presentó 1.530 casos de lesiones por accidentes de tránsito de los cuales los hombres representaron el $57,9 \%(7)$.

Actualmente, no se conoce el impacto de los AT, debido a la complejidad del problema y a las distintas causas que no se han podido determinar. Para tener una aproximación actualizada sobre el impacto de los AT en la población de Pereira es necesario tener en cuenta todos los factores relacionados a la ocurrencia del evento, desde parámetros estadísticos hasta indicadores de morbimortalidad asociados a la carga de la enfermedad.

La salud pública se convierte en un compromiso de la sociedad, como eje fundamental de salud e integralidad. El Plan Decenal de Salud Pública (PDSP), 2012-2021 de Colombia, establece un pacto social y un mandato ciudadano, que articula múltiples actores y sectores públicos, privados y comunitarios para crear condiciones que garanticen el bienestar integral y la calidad de vida de los $\operatorname{colombianos}^{(8)}$. 
El proyecto se encamina a la construcción del objetivo del PDSP de mantener cero tolerancia frente a la mortalidad, la morbilidad y la discapacidad evitables, convirtiéndose en uno de los grandes retos del PDSP, 2012-2021, el cual aborda el concepto de salud como: "el resultado de la interacción armónica de las condiciones biológicas, mentales, sociales y culturales del individuo, así como con su entorno y con la sociedad, a fin de poder acceder a un mejor nivel de

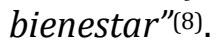

El Plan de Desarrollo Municipal 2016-2019 de la ciudad de Pereira(9), enfoca su objetivo general a la persona humana como el centro de todos los propósitos de la política pública. Dentro de éste se encuentra el eje de desarrollo social y la reconciliación, el cual requiere el mejoramiento de las condiciones de salud de todos los habitantes a través de un plan de atención básico en salud, que permita, entre otros, controlar al máximo las muertes evitables, como en este caso las causadas por accidentes de tránsito. De acuerdo a la magnitud de este creciente problema, se planteó este estudio con el objetivo de caracterizar epidemiológicamente las lesiones por accidentes de tránsito reportados en personas atendidas en una institución de salud en el municipio de Pereira entre 2014 y 2017.

\section{Materiales y métodos}

Estudio de tipo descriptivo y transversal basado en fuentes secundarias de información desagregadas según ciertas características sociodemográficas, epidemiológicas y clínicas de los accidentes de tránsito reportados por el Seguro Obligatorio de Accidentes de Tránsito (SOAT) y atendidos en una entidad de salud del municipio de Pereira, durante el período comprendido entre los años 2014 a 2017. La fuente de información fue la epicrisis hospitalaria o informe de accidente de tránsito derivada del registro de accidentes del sistema de Vigilancia Epidemiológica de las Lesiones de Causa Externa de la entidad de salud. Se incluyeron todos los accidentes en donde se registren una o varias personas lesionadas.
El universo de esta investigación se basa en los registros institucionales de pacientes atendidos como consecuencia de accidentales de tránsito en la entidad de salud. La población de estudio está constituida por todas las muertes y lesiones de causa externa fatal o no fatal, consecuencia de accidentes de tránsito, cuyo hecho haya ocurrido entre los años 2014 a 2017. Se incluyeron todos los accidentes de tráfico de vehículos de motor con código CIE10: V02-V04, V09.0, V09.3-V09.9, V12.3-V14.9, V19.4-V19.6, V20.0-V28.3-V28.9, V29.4-V79.9, V80.3-V80.5, V81.0-V81.1, V82.0V82.1, V83-V86, V87.0-V87.8, V88.0-V88.8, V89.0, V89.2, V89.9, Y85.0. De igual manera los traumatismos reportados por el SOAT con los códigos: S00-S09, S10-S19, S20-S29, S30-S39, S40-S49, S50-S59, S60-S79, S80-S89, S90-S99, T00-T07, T08-T14, que fueron solamente registrados bajo código de lesión o traumatismo y no de accidente de tránsito.

Debido a la sistematización de la historia clínica pero no de la base de datos, se pudo totalizar un total de 15.000 accidentes de tránsito que fueron registrados en la entidad de salud propuesta para este estudio durante el período mencionado, por lo cual fue necesario realizar un proceso de muestreo. Para una prevalencia esperada del $50 \%$, un nivel de confiabilidad del $95 \%$, un efecto del diseño del $1 \%$ en una población de menos de 100.000 habitantes, el cálculo del tamaño de la muestra se realizó mediante la fórmula para hallar una proporción en poblaciones finitas(10). Esperando un porcentaje de no respuesta del $20 \%$, se determinó un tamaño de muestra final de 460 registros. El cálculo del tamaño de muestra fue realizado en OpenEpi, versión 3. Se utilizó el software Epi-dat para la selección aleatoria de la muestra mediante un Muestreo Aleatorio Simple sin reemplazo.

Dentro de los criterios de inclusión se tuvieron la fecha del hecho, el cual debería estar entre el $1^{\circ}$ de enero de 2014 y el 30 de junio de 2017, y los casos reportados por las aseguradoras de accidentes de tránsito atendidos en la institución de salud: ser peatón, conductor o integrante del vehículo. Se excluyeron aquellos casos que una vez ingresados a la institución de salud se hayan remitido dentro 
de las primeras 6 horas de ocurrido el accidente sin haber recibido atención o tratamiento.

Las variables sociodemográficas analizadas fueron: sexo, edad, lugar de nacimiento, lugar de residencia, afiliación a seguridad social y estado civil. Dentro de las variables directas del estudio en relación al accidente de tránsito se utilizó el tipo de accidente, la condición de la víctima (peatón, motociclista, pasajero, ciclista, conductor, parrillero), el tipo de lesión (laceración, luxación, fractura, contusión), hospitalización (sí, no), días de hospitalización y el sitio anatómico de lesión el cual fue clasificado según códigos CIE-10. Se empleó una categoría cualitativa que se denominó "Causa que provocó el accidente", se recolectó la información de manera textual según el reporte del médico de urgencias.

La recolección de la información se llevó a cabo mediante los registros suministrados en las historias clínicas, en una hoja de cálculo de EXCEL ${ }^{\circledR}$ que incluía las variables anteriormente mencionadas. La información se manejó de manera estricta y confidencial, a cada formato de recolección se le asignó un código con secuencia numérica.

Se realizó un análisis descriptivo univariado, de cada una de las variables de estudio. Se identificó y clasificó los accidentes reportados por el SOAT con los códigos CIE-10. Posteriormente, a las variables de naturaleza cuantitativa se les aplicó la prueba de Kolmogorov-Smirnov para verificar el supuesto de normalidad, y de acuerdo con su distribución se presentaron las medias de tendencia central y de dispersión pertinentes con sus correspondientes intervalos de confianza al $95 \%$.

Para las variables de tipo nominal $\mathrm{u}$ ordinal se calcularon frecuencias y proporciones; estos análisis se complementaron con tablas cruzadas en las cuales se usaron pruebas estadísticas paramétricas como la prueba $t$ de student $\mathrm{y}$ análisis de varianza y no paramétrica como Chicuadrado y Kruskal Wallis, a un nivel de significancia del $5 \%$. Para la variable causa que provocó el accidente de tránsito, que se recolectó de forma textual, como es descrita en la historia clínica, se realizó un análisis categorías para lograr identificar una tendencia en las causas que producen los accidentes de tránsito. Se utilizó el paquete estadístico SPSS Versión 23.

\section{Consideraciones éticas}

Esta propuesta de investigación fue aprobada por el Comité de Investigación de la Fundación Universitaria del Área Andina. Se considera como un estudio sin riesgo según la Resolución № 008430 de 1993, que establece las normas científicas, técnicas y administrativas para la investigación en salud. Se contó con la aprobación de la institución de salud para la realización de este estudio en fuentes de datos secundarios, al ser un estudio que no involucró directamente a los pacientes sino a historias clínicas y registros.

\section{Resultados}

Se analizaron 460 historias clínicas de casos reportados por el SOAT, atendidos en la institución de salud, casos que corresponden a la muestra seleccionada del universo de estudio. No se registraron pérdidas de datos correspondientes a las variables principales del estudio. Dentro del estudio se pudo encontrar la variable edad con una mediana de 30 años y un rango intercuartílico de 20.

En la Tabla 1, se puede apreciar que el $64,1 \%$ de los lesionados corresponde a hombres. El 86,5\% de los casos estaban reportados en las historias clínicas por el SOAT, mientras que el 13,5\%, se encontraban asignados a los regímenes de salud, aun cuando fueron producto de accidentes de tránsito. El 90,9\% de la muestra, eran solteros y un $7,6 \%$ casados. 
Tabla 1. Caracterización de los accidentes de tránsito de la población de estudio. 2014-2017

\begin{tabular}{|c|c|c|c|}
\hline & & $\mathbf{N}^{\circ}$ casos & $\%$ \\
\hline \multirow[t]{2}{*}{ Sexo } & Hombre & 295 & 64,1 \\
\hline & Mujer & 165 & 35,9 \\
\hline \multirow{4}{*}{$\begin{array}{l}\text { Procedencia-Régimen } \\
\text { de Salud* }\end{array}$} & SOAT & 398 & 86,5 \\
\hline & Subsidiado & 39 & 8,5 \\
\hline & Contributivo & 19 & 4,1 \\
\hline & Especial & 4 & 0,9 \\
\hline \multirow[t]{4}{*}{ Estado civil } & Soltero & 418 & 90,9 \\
\hline & Casado/unión libre & 35 & 7,6 \\
\hline & Viudo & 6 & 1,3 \\
\hline & Separado & 1 & 0,2 \\
\hline \multirow[t]{5}{*}{ Tipo de accidente } & Caída & 198 & 43,0 \\
\hline & Atropellamiento & 118 & 25,7 \\
\hline & Colisión & 82 & 17,8 \\
\hline & Choque & 55 & 12,0 \\
\hline & Volcadura & 7 & 1,5 \\
\hline \multirow{7}{*}{$\begin{array}{l}\text { Causa que provocó el } \\
\text { accidente }\end{array}$} & Impericia del conductor & 181 & 39,3 \\
\hline & Caída espontanea & 155 & 33,7 \\
\hline & Distracción & 93 & 20,2 \\
\hline & Vehículo fantasma & 34 & 7,4 \\
\hline & Alcoholismo & 16 & 3,5 \\
\hline & Exceso de velocidad & 4 & 0,9 \\
\hline & Otras causas & 45 & 1,1 \\
\hline \multirow{7}{*}{$\begin{array}{l}\text { Condición de la } \\
\text { víctima }\end{array}$} & Motociclista & 204 & 44,3 \\
\hline & Peatón & 109 & 23,7 \\
\hline & Parrillero & 74 & 16,1 \\
\hline & Pasajero & 29 & 6,3 \\
\hline & Ciclista & 23 & 5,0 \\
\hline & Conductor & 11 & 2,4 \\
\hline & Sin dato & 10 & 2,2 \\
\hline \multirow[t]{4}{*}{ Tipo de lesión } & Contusión & 296 & 64,3 \\
\hline & Fractura & 96 & 20,9 \\
\hline & Laceración & 55 & 12,0 \\
\hline & Luxación & 13 & 2,8 \\
\hline \multirow[t]{2}{*}{ Hospitalización } & $\mathrm{Si}$ & 230 & 50,0 \\
\hline & No & 230 & 50 , \\
\hline \multirow[t]{2}{*}{ Lesiones múltiples } & $\mathrm{Si}$ & 143 & 31,1 \\
\hline & No & 143 & 68,9 \\
\hline
\end{tabular}

Al momento de caracterizar los accidentes de tránsito sufridos por la población objeto de estudio, se encontró que el $43 \%$ de los casos fueron representados por caídas, seguido con un $25,7 \%$ de atropellamientos donde se encontraba vinculado un peatón, en casi todas las ocasiones. La colisión representó el $17,8 \%$, seguido muy cerca por el choque con un $12 \%$, la volcadura fue del $1,5 \%$.

Dentro de las causas que provocaron los accidentes, obtenidas textualmente de las historias clínicas y del reporte otorgado por el médico de urgencias. Se logró establecer 7 categorías que describen las posibles causas asociadas al accidente y a la lesión sufrida por la unidad de análisis. La categoría con mayor porcentaje fue la impericia del conductor con un $39,3 \%$, seguido por la caída espontánea con un $33,7 \%$, el cual era referido por el paciente al momento del ingreso a urgencias. La distracción tanto del peatón, como del conductor fue del $20,2 \%$. Los accidentes producidos por los vehículos fantasma según el lesionado, fue del $7,4 \%$. El alcoholismo y el exceso de velocidad se encuentran por debajo del $10 \%$ al igual que otras causas identificadas diferentes a las categorías establecidas. 
La condición de la víctima se vio reflejada por el $44,3 \%$ de motociclistas con el mayor porcentaje de accidentados dentro de la población de estudio. El peatón representó el 23,7\%, muy cercano a lo presentado por parrillero, quien es el acompañante o pasajero de los vehículos de dos ruedas con un 16,1\%. El pasajero, ciclista y conductor de vehículo se encuentra con un porcentaje inferior al 10\%. En los tipos de lesión el más frecuente, fue la contusión con un $64,3 \%$, seguido por las fracturas con un $20,9 \%$, las laceraciones con un $12 \%$ y las luxaciones con un $2,8 \%$.

El $50 \%$ de los lesionados fueron hospitalizados con una mediana de 1 día y un rango intercuartílico de 2. El 31,1\% de la muestra, sufrió más de una lesión en distintas partes del cuerpo.

En la Figura 1 se caracterizan las lesiones ocasionadas por los accidentes, en la vista anterior se observa que las extremidades inferiores sufrieron el 27,8\% de las lesiones, muy similar a lo presentado en las extremidades superiores con un $25,2 \%$.

El $18 \%$ de las lesiones ocurrieron en cabeza y cráneo. Las lesiones de la región torácica fueron del 8,3\%, la región pélvica y la articulación de la cadera fue del 5,2\%. La región cervical, torácica y lumbar posterior, la región abdominal, los genitales presentaron una prevalencia baja, su interés clínico en relación a la anatomía convierte dichas lesiones en relevantes. Es de resaltar que se presentó un caso de lesión abdominal, pero fue reportado y discriminado como trauma obstétrico.

En la Tabla 2 se puede ver el análisis de la variable edad asociada con distintas variables cualitativas de importancia clínica relacionadas al accidente de tránsito.

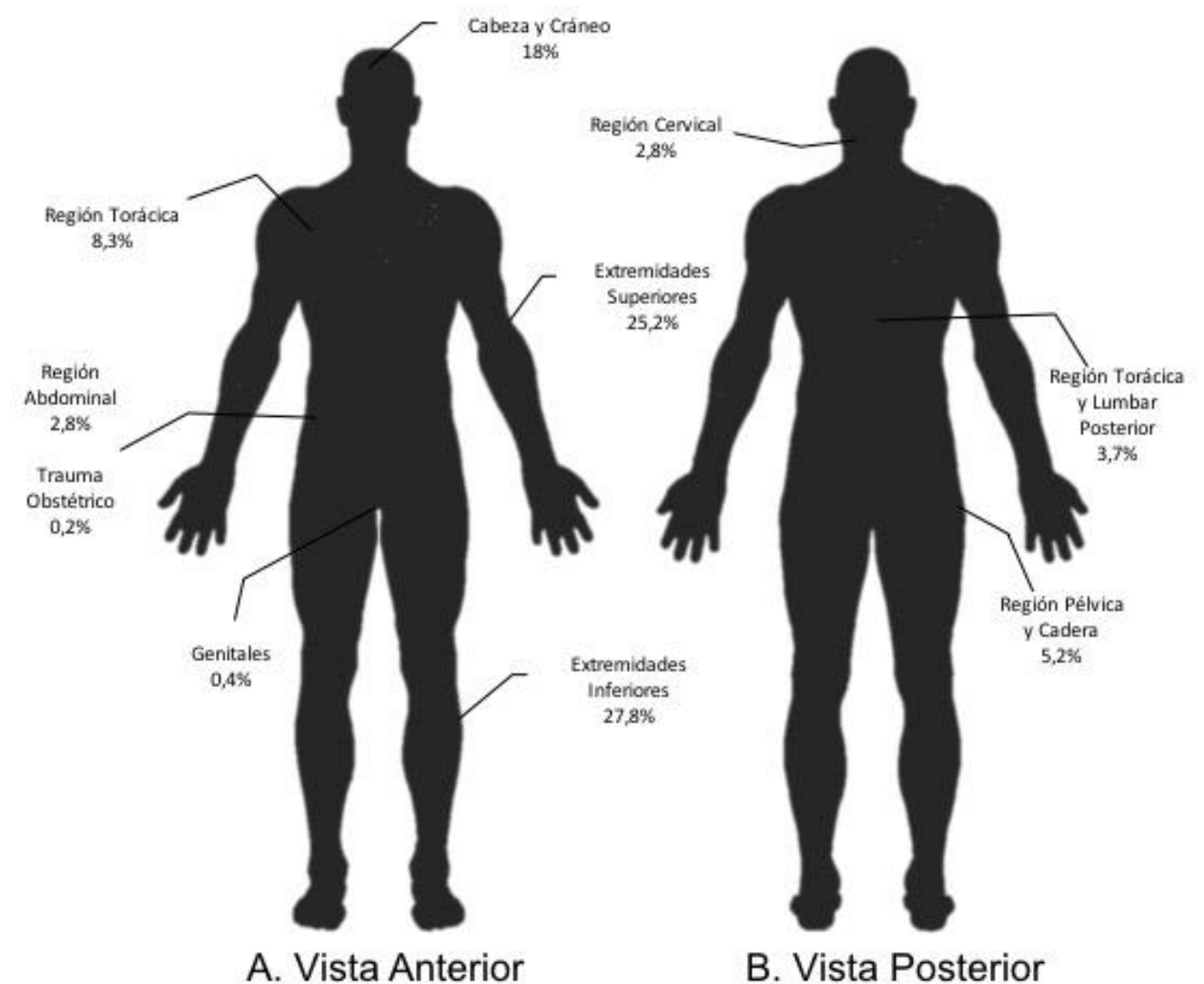

Figura 1. Distribución de las lesiones por accidentes de tránsito 
Tabla 2. Asociación entre la edad y las distintas variables clínicas y del accidente de tránsito

\begin{tabular}{|c|c|c|c|}
\hline & & \multicolumn{2}{|c|}{ Edad } \\
\hline & & $\begin{array}{c}\text { Mediana } \\
\text { (RIQ) }\end{array}$ & Valor P \\
\hline \multirow[t]{2}{*}{ Sexo } & Hombre & $30(19)$ & 0,489 \\
\hline & Mujer & $31(22)$ & \\
\hline \multirow[t]{5}{*}{ Tipo de accidente } & Caída & $31(18)$ & 0,311 \\
\hline & Atropellamiento & $30,5(30)$ & \\
\hline & Colisión & $30(16)$ & \\
\hline & Choque & $27(19)$ & \\
\hline & Volcadura & $41(51)$ & \\
\hline \multirow{6}{*}{$\begin{array}{l}\text { Causa que provocó el } \\
\text { accidente }\end{array}$} & Impericia del conductor & $30(18)$ & 0,063 \\
\hline & Caída espontánea & $32(22)$ & \\
\hline & Distracción & $24(28)$ & \\
\hline & Vehículo fantasma & $43,5(32)$ & \\
\hline & Alcoholismo & $26,5(32)$ & \\
\hline & Otras causas & $31(11)$ & \\
\hline \multirow{6}{*}{$\begin{array}{l}\text { Condición de la } \\
\text { víctima }\end{array}$} & Motociclista & $30(15)$ & $0,047^{*}$ \\
\hline & Peatón & $32(329$ & \\
\hline & Parrillero & $29,5(26)$ & \\
\hline & Pasajero & $41(30)$ & \\
\hline & Ciclista & $25(20)$ & \\
\hline & Conductor & $37(26)$ & \\
\hline \multirow[t]{4}{*}{ Tipo de lesión } & Contusión & $30(20)$ & 0,689 \\
\hline & Fractura & 28(19) & \\
\hline & Laceración & $31(16)$ & \\
\hline & Luxación & $23(31)$ & \\
\hline \multirow[t]{2}{*}{ Hospitalización } & $\mathrm{Si}$ & $30(20)$ & 0,99 \\
\hline & No & $30(19)$ & \\
\hline \multirow[t]{2}{*}{ Lesiones múltiples } & $\mathrm{Si}$ & $26(18)$ & $0,046^{*}$ \\
\hline & No & $32(21)$ & \\
\hline \multirow[t]{8}{*}{ Sitio afectado } & Extremidades inferiores & $27(21)$ & $<0,0001^{*}$ \\
\hline & Extremidades superiores & $34(18)$ & \\
\hline & Cabeza y cráneo & $25(18)$ & \\
\hline & Región torácica & $33(24)$ & \\
\hline & Región pélvica y cadera & $40(21)$ & \\
\hline & Región torácica y lumbar posterior & $28(12)$ & \\
\hline & Región abdominal & $32(20)$ & \\
\hline & Región cervical & $26(14)$ & \\
\hline
\end{tabular}

*Prueba de Kruskall Walls

No se encontró diferencias significativas entre la edad y el sexo, el tipo de accidente, la causa del accidente, el tipo de lesión, hospitalización. Se pudo establecer diferencias significativas entre las medianas de las edades en las variables de condición de la víctima con un valor $\mathrm{p}=0,047$, donde los motociclistas quienes fueron los lesionados con mayor porcentaje presentaron una mediana de 30 años (RIQ=15). La edad y las lesiones múltiples presentadas por los sujetos de estudio presentaron diferencias significativas con un valor $p=0,046$. El sitio anatómico afectado tuvo un valor p=0,0001, encontrándose diferencias significativas entre la edad y el sitio de la lesión.

En la Tabla 3 se puede observar la distribución porcentual de los distintos tipos de lesión. Todas las variables presentadas a excepción de hospitalización, presentaron valores $\mathrm{p}<0,005$, encontrándose diferencias significativas entre dichas variables. El 64,6\% de las fracturas, fueron en hombres y de similar proporción para los demás tipos de lesión, con un valor p= 0,002. 
Tabla 3. Distribución porcentajes de los tipos de lesión y distintas variables asociadas al accidente de tránsito

\begin{tabular}{|c|c|c|c|c|c|c|}
\hline & & \multicolumn{4}{|c|}{ Tipo de Lesión } & \multirow[t]{2}{*}{ Valor $\mathbf{p}$} \\
\hline & & $\begin{array}{c}\text { Laceración } \\
n(\%)\end{array}$ & $\begin{array}{c}\text { Luxación } \\
n(\%)\end{array}$ & $\begin{array}{c}\text { Fractura } \\
n(\%)\end{array}$ & $\begin{array}{c}\text { Contusión } \\
n(\%)\end{array}$ & \\
\hline \multirow[t]{2}{*}{ Sexo } & Hombre & $42(76,4)$ & $13(100)$ & $62(64,6)$ & $178(60,1)$ & $0,001^{* *}$ \\
\hline & Mujer & $13(23,6)$ & $0(0,0 \%)$ & $34(35,4)$ & $118(39,9)$ & \\
\hline \multirow{5}{*}{$\begin{array}{l}\text { Tipo de } \\
\text { accidente }\end{array}$} & Caída & $30(54,5)$ & $10(76,9)$ & $46(47,9)$ & $112(37,8)$ & $0,002^{* *}$ \\
\hline & Atropellamiento & $13(23,6)$ & $2(15,4)$ & $27(28,1)$ & $76(25,7)$ & \\
\hline & Colisión & $9(16,4)$ & $0(0,0)$ & $10(10,4)$ & $63(21,3)$ & \\
\hline & Choque & $3(5,5)$ & $0(0,0)$ & $13(13,5)$ & $39(13,2)$ & \\
\hline & Volcadura & $0(0,0)$ & $1(7,7)$ & $0(0,0)$ & $6(2,0)$ & \\
\hline \multirow{7}{*}{$\begin{array}{l}\text { Condición de la } \\
\text { victima }\end{array}$} & Motociclista & $27(49,1)$ & $10(76,9)$ & $42(43,9)$ & $125(42,2)$ & $0,004^{* *}$ \\
\hline & Peatón & $12(21,8)$ & $2(15,4)$ & $23(24,0)$ & $72(24,3)$ & \\
\hline & Parrillero & $13(23,6)$ & $0(0,0)$ & $15(15,6)$ & $46(15,5)$ & \\
\hline & Pasajero & $0(0,0)$ & $0(0,0)$ & $4(4,2)$ & $25(8,4)$ & \\
\hline & Ciclista & $1(1,8)$ & $0(0,0)$ & $8(8,3)$ & $14(4,7)$ & \\
\hline & Conductor & $0(0,0)$ & $1(7,7)$ & $0(0,0)$ & $10(3,4)$ & \\
\hline & Sin dato & $2(3,6)$ & $0(0,0)$ & $4(4,2)$ & $4(1,4)$ & \\
\hline \multirow[t]{2}{*}{ Hospitalización } & $\mathrm{Si}$ & $20(36,4)$ & $6(46,2)$ & $59(61,5)$ & $145(49)$ & $0,25^{*}$ \\
\hline & No & $35(63,6)$ & $7(53,8)$ & $37(38,5)$ & $151(51,0)$ & \\
\hline \multirow{2}{*}{$\begin{array}{l}\text { Lesiones } \\
\text { múltiples }\end{array}$} & $\mathrm{Si}$ & $20(36,4)$ & $1(7,7)$ & $23(24,0)$ & $99(33,4)$ & $0,045^{* *}$ \\
\hline & No & $35(63,6)$ & $12(92,3)$ & $73(76,0)$ & $197(66,6)$ & \\
\hline \multirow[t]{10}{*}{ Sitio afectado } & Extremidades inferiores & $24(43,6)$ & $3(23,1)$ & $28(29,2)$ & $73(24,7)$ & $<0,000^{* *}$ \\
\hline & Extremidades superiores & $14(25,5)$ & $8(61,5)$ & $42(43,8)$ & $52(17,6)$ & \\
\hline & Cabeza y cráneo & $12(21,8)$ & $0(0,0)$ & $14(14,6)$ & $57(19,3)$ & \\
\hline & Región torácica & $1(1,8)$ & $0(0,0)$ & $6(6,3)$ & $31(10,5)$ & \\
\hline & Región pélvica y cadera & $0(0,0)$ & $0(0,0)$ & $0(0,0)$ & $23(8,1)$ & \\
\hline & $\begin{array}{l}\text { Región torácica y lumbar } \\
\text { posterior }\end{array}$ & $0(0,0)$ & $0(0,0)$ & $4(4,2)$ & $13(4,4)$ & \\
\hline & Región abdominal & $0(0,0)$ & $0(0,0)$ & $0(0,0)$ & $13(4,4)$ & \\
\hline & Región cervical & & & & & \\
\hline & Trauma obstétrico & $0(0,0)$ & $0(0,0)$ & $0(0,0)$ & $1(0,3)$ & \\
\hline & No especificado & $3(3,6)$ & $0(0,0)$ & $0(0,0)$ & $23(7,8)$ & \\
\hline
\end{tabular}

*Prueba de Chi-cuadrado

** Prueba de verosimilitud

El tipo de accidente en relación a la lesión, fue representado por las caídas con un $37,8 \%$ de las contusiones. En los atropellamientos el $28,1 \%$ fueron fracturas. La colisión $(21,3 \%)$ y el choque $(13,2 \%)$ presentaron el mayor porcentaje para las contusiones. El valor para el tipo de accidente fue de $\mathrm{p}=0,002$.

Dentro de las condiciones de la víctima, los motociclistas sufrieron el $42,2 \%$ de las contusiones, el 43,9\% de las fracturas, el 76,9\% de las luxaciones y el 49,1\% de las laceraciones. Los peatones presentaron el $24,3 \%$ de las contusiones, dato similar a las fracturas y a las lesiones en parrilleros. De las personas que obtuvieron múltiples lesiones, el $36,4 \%$ presentaron laceraciones, el 33,4\% contusiones, y el $24 \%$ fracturas, encontrándose diferencias significativas con un valor $\mathrm{p}=0,045$ (Tabla 3).

En relación al sitio afectado y el tipo de lesión el $43,6 \%$ de las laceraciones se presentaron en miembros inferiores, un $25,5 \%$ en las extremidades superiores, y el $21,8 \%$ en cabeza y cráneo. El $23,1 \%$ de las luxaciones fueron presentadas en las extremidades inferiores, el $61,5 \%$ en las extremidades superiores. El 29,2\% de las fracturas se encontraron en las extremidades inferiores, el $43,8 \%$ en las extremidades superiores y el $14,6 \%$ en la cabeza y cráneo. Para la contusión el mayor porcentaje se presentó en las extremidades inferiores con un $24 \%$. 


\section{Discusión}

Se encontró 460 casos de accidentes de tránsito que fueron atendidos en la institución de salud en el periodo 2014- 2017, en su gran mayoría fueron hombres, con una mediana de edad de 30 años. Para este caso, su estado civil en gran proporción fue soltero y transitaban en motocicleta durante el accidente. Gran parte de los casos fueron reportados por la aseguradora de accidentes de tránsito. La caída como tipo de accidente fue el que tuvo el mayor incremento asociado al tipo de lesión más común, que fue la contusión, seguido por las fracturas. La mitad de la muestra estuvo hospitalizada con una mediana de 1 día, acompañado de una alta prevalencia de múltiples lesiones. Según el sitio anatómico, las lesiones que más se presentaron fueron en las extremidades.

Se resalta en el presente estudio diferentes características involucradas en las lesiones producidas por los accidentes de tránsito. El sexo masculino y el estado civil soltero predominó al igual que en otros estudios publicados(11,12). Sandra Porras, et al., en un estudio realizado con motociclistas en Medellín, Colombia, encontraron una prevalencia del $50 \%$ en los hombres, quienes tenían una media de edad de 40 años o menos, similar a lo presentado en este estudio(13).

Por su parte en India, país que posee uno de los índices más altos de accidentes de tránsito en el mundo, el $83,5 \%$ de las víctimas fueron hombres(3); se estableció que uno de los grupos más vulnerables, fueron los peatones, datos posibles de contrastar en el presente estudio, en el cual se encontró una alta tasa de accidentalidad, donde el involucrado es un peatón. De esta manera se evidencia que el peatón está en situación de vulnerabilidad frente a otros usuarios de carretera involucrados en lesiones por accidente de tránsito(3).

Al comparar por sexo, los hombres son los que más se accidentan en motocicletas; posibles explicaciones radican en que ellos son quienes más eligen este medio de transporte y de desplazamiento a sitio de trabajo, además en gran proporción no poseen la percepción del riesgo, frente al uso de la alta velocidad y conducir ebrios, hasta llevarlos a la muerte(14). Sin embargo, actualmente no se conoce bien los motivos de esta asociación, se han planteado diversas hipótesis en comparación con las mujeres, los peatones masculinos están involucrados en colisiones de mayor severidad intrínseca. Por ejemplo, se ha informado de colisiones nocturnas en las que los hombres pueden verse involucrados con mayor frecuencia, porque las mujeres tienden a caminar o transitar menos en horas nocturnas y en muchas ocasiones estas colisiones son más severas que las que ocurren durante el día(15,16).

En este estudio fue posible corroborar que muchas de las causas de los accidentes de tránsito también se ven asociadas a la distracción tanto del conductor como de los peatones, cuando estos se ven involucrados; a este factor se podría sumar la imprudencia e impericia de dichos conductores que representa una de las principales causas de los accidentes, como se evidencia en un estudio realizado en Perú(17).

Las lesiones causadas por los accidentes de tránsito dependen específicamente de una biomecánica, la cual va ligada a la velocidad y a la cantidad de energía que se imparta desde el vehículo en movimiento. En el atropellamiento automovilístico como uno de los tipos de accidente más frecuente, uno de los principales afectados es el peatón, que en gran proporción sufre las lesiones en la parte inferior del cuerpo(18,19). Dato que se encuentra relacionado con lo encontrado en el presente estudio, donde las lesiones en los miembros inferiores obtuvieron una representatividad significativa. Las lesiones como las contusiones y laceraciones están asociadas a una fase de arrastre donde el peatón se desplaza junto con el vehículo y empiezan a presentarse las lesiones múltiples.

Según el tipo de lesión, se puede observar un gran porcentaje de traumas múltiples, similar a lo encontrado por Cardona, et al., sin embargo, se tiene pocas similitudes con el lugar anatómico de la lesión, donde el trauma encefalocraneano fue del $36 \%(20)$, al igual que en el estudio realizado en Cienfuegos(21) con ciclistas involucrados en accidentes de tránsito; mientras que en el estudio realizado en Sao Paulo-Brasil(22), las lesiones en 
las extremidades inferiores y superiores alcanzaron el $60 \%$, seguido de los traumas craneanos con un $31 \%$, en concordancias con este estudio y con el realizado en el Hospital Nacional Itaguá, Paraguay(23).

En este tipo de transporte se obtiene más probabilidad de causar lesiones graves o la muerte. Los accidentes en que se encuentra involucrada una sola motocicleta son causados principalmente por el exceso de velocidad o por el consumo de alcohol(5,6,24,25). Al momento de presentarse un accidente de tránsito donde se encuentra involucrado un objeto fijo, las motocicletas tienen poca capacidad de absorber energía, por lo que el conductor, pasajero o parrillero, recibe toda la cantidad del impacto, generando un gran daño tisular y sistémico. Estas corren mayores peligros asociados al estado de las carreteras, más que otros automóviles. Por la naturaleza de este medio de transporte, son máquinas más inestables, que, frente a condiciones del pavimento como baches, desniveles, objetos y personas inesperadas, se convierten en una amenaza para el conductor; cuando las motocicletas se estrellan el conductor no posee la suficiente protección de un vehículo cerrado $(3,4,26)$.

Es preciso establecer que los accidentes de tránsito siguen tomando fuerza, como una problemática en salud pública. Se podría investigar la relación entre el número de automotores y motocicletas y la alta tasa de accidentes de tránsito, considerando como un evento de importancia y atención dentro de las estadísticas de las lesiones personales no fatales. Analizar el contexto de los accidentes de tránsito desde un enfoque cualitativo, permitirá obtener nuevos análisis frente a la percepción del riesgo, y el control que se tiene sobre los diferentes medios de transporte que se poseen. De igual manera, plantear una estrategia para la sensibilización frente al exceso de velocidad, consumo de alcohol o la impericia del conductor. Dichas acciones no van destinadas sólo a la reducción de las lesiones por accidentes de tránsito, sino a mitigar el riesgo de la mortalidad de dichos vehículos de transporte(27).
Con relación a las limitaciones del estudio, se presentaron algunas dificultades relacionadas con el diligenciamiento de los sistemas de información y la reducida caracterización sociodemográfica que contienen muchas de las historias clínicas de los pacientes. Por ejemplo, las variables ocupación y nivel educativo, no se obtuvieron por no ser encontradas en las historias clínicas. Además, los diagnósticos y sitios de lesión no especificados limitan el análisis exhaustivo de los datos. Para futuras investigaciones se recomienda analizar indicadores epidemiológicos, realizar análisis de factores de riesgo y estudios de tendencias temporales, con el fin de poder orientar mejor las acciones de intervención.

\section{Conclusiones}

Los hombres fueron la población con mayor proporción de lesionados, estas lesiones son principalmente, derivadas de los accidentes en motocicleta y peatones, asociados a la impericia del conductor o a la distracción del peatón. Estos desencadenaron en mayor proporción en contusiones y fracturas; afectando la productividad e incrementando la carga de la enfermedad. Dicha información debe contribuir al fortalecimiento de políticas viales tanto municipales como nacionales.

\section{Financiación}

No existen fuentes de financiación diferentes a la Fundación Universitaria del Área Andina, Seccional Pereira

\section{Agradecimientos}

Agradecimientos al Hospital Universitario San Jorge, a los auxiliares de Investigación Lina Barrera, Michelle Molina y al docente Investigador German Acevedo de la Fundación Universitaria del Área Andina, Seccional Pereira. Este artículo es derivado del trabajo de investigación como requisito de grado en la Maestría en Salud Pública y Desarrollo Social de la Fundación Universitaria del Área Andina de los 4 primeros autores. 


\section{Conflicto de intereses}

No se declaran conflictos de intereses con relación a esta investigación.

\section{Referencias}

1. Organización Mundial de la Salud. Informe sobre la situación mundial de la seguridad vial 2015. Ginebra: OMS; 2015.

2. Organización Mundial de la Salud. Sistema de datos: Manual de Seguridad vial para Decisores y Profesionales. Ginebra: OMS; 2010.

3. Bayan P, Bhawalkar JS, Jadhav SL, Banerjee A. Profile of non-fatal injuries due to road traffic accidents from a industrial town in India. Int J Crit Illn Inj Sci. 2013;3(1):8-11. DOI: 10.4103/2229-5151.109409

4. Herman J, Ameratunga S, Jackson R. Burden of road traffic injuries and related risk factors in low and middle-income Pacific Island countries and territories: a systematic review of the scientific literature (TRIP 5). BMC Public Health. 2012;12:479. DOI: 10.1186/14712458-12-479

5. Barberia E, Suelves JM, Xifro A, Medallo J. [Differences between immediate and 30-day deaths due to traffic injuries according to forensic sources]. Gac Sanit. 2015;29(Suppl1):66-9.

DOI: 10.1016/j.gaceta.2015.04.012

6. Al-Abdallat IM, Al Ali R, Hudaib AA, Salameh GA, Salameh RJ, Idhair AK. The prevalence of alcohol and psychotropic drugs in fatalities of road-traffic accidents in Jordan during 2008-2014. J Forensic Leg Med. 2016;39:130-4. DOI: 10.1016/j.jflm.2016.01.018

7. Instituto Nacional de Medicina Legal y Ciencias Forenses. Forensis: Datos para la vida. Bogotá: Instituto Nacional de Medicina Legal y Ciencias Forenses 2017.

8. Ministerio de Salud y Protección Social. Plan Decenal de Salud Pública, PDSP, 2012-2021. Bogotá: Ministerio de Salud Pública; 2012.

9. Alcaldía de Pereira. Plan de Desarrollo Municipal 20162019: "Pereira, capital del eje". Pereira: Alcaldía Municipal; 2016

10. Silva LC. Diseño razonado de muestras y captación de datos para la investigación sanitaria. Madrid: Díaz de Santos; 2000.

11. Ruiz JI, Herrera AN. Accidentes de tránsito con heridos en Colombia según fuentes de información: caracterización general y tipologías de accidentes. Ces Psicol. 2016;9(1):32-46. DOI: 10.21615/cesp.9.1.3

12. Shavaleh R, Motevalian SA, Mahdavi N, Haddadi M, Mohaghegh MR, Hamedi Z. Epidemiological study of hospitalized road traffic injuries in Iran 2011. Med J Islam Repub Iran. 2018;32:50. DOI: 10.14196/mjiri.32.50

13. Porras S, Grisales H. Años potenciales de vida perdidos por incidentes viales de motociclistas, Medellín, 20092012: un analisis espacial por sitio de la ocurrencia. Rev Méd Risaralda. 2017;23(1):22-9. DOI: $10.22517 / 25395203.13881$

14. de Carvalho HB, Andreuccetti G, Rezende MR, Bernini C, Silva JS, Leyton $\mathrm{V}$, et al. Alcohol and drug involvement in motorcycle driver injuries in the city of Sao Paulo, Brazil: Analysis of crash culpability and other associated factors. Drug Alcohol Depend. 2016;162:199-205. DOI: 10.1016/j.drugalcdep.2016.03.007

15. Cordellieri P, Baralla F, Ferlazzo F, Sgalla R, Piccardi L, Giannini AM. Gender Effects in Young Road Users on Road Safety Attitudes, Behaviors and Risk Perception. Front Psychol. 2016;7:1412. DOI: 10.3389/fpsyg.2016.01412

16. Onieva-Garcia MA, Martinez-Ruiz V, Lardelli-Claret $P$, Jimenez-Moleon JJ, Amezcua-Prieto C, de Dios Luna-DelCastillo J, et al. Gender and age differences in components of traffic-related pedestrian death rates: exposure, risk of crash and fatality rate. Inj Epidemiol. 2016;3:14. DOI: 10.1186/s40621-016-0079-2

17. Choquehuanca-Vilca V, Cardenas-Garcia F, CollazosCarhuay J, Mendoza-Valladolid W. [Epidemiological profile of road traffic accidents in Peru, 2005-2009]. Rev Peru Med Exp Salud Pública. 2010;27(2):162-9.

18. Ramírez Muñoz JE. Accidentes de tránsito terrestre. Med leg Costa Rica. 2013;30(2):78-85.

19. Segura Cardona AM, Cardona Arango D, Berbesí FernándezI DY, Agudelo Martínez A. Mortalidad por accidente de tránsito en el adulto mayor en Colombia. Rev de Saúde Pública. 2017;51(21):1-8. DOI: 10.1590/s1518-8787.2017051006405

20. Cardona-Arbeláez SA, Molina-Castaño CF, Arango-Álzate CM, Pichott-Padilla JT. Caracterización de accidentes de tránsito y valoración tarifaria de la atención médica en el servicio de urgencias, Caldas-Antioquia 2007-2008. Rev Gerenc Polit Salud. 2010;9(19):216-28.

21. Jorge Miguez A, Godoy del Sol H, Ortis Sagasta M. Caracterización de la mortalidad por accidentes de tránsito con participación de ciclos: un problema sociomédico. MediSur. 2010;8(4):57-62.

22. Gawryszewski VP, Coelho HM, Scarpelini S, Zan R, Jorge $\mathrm{MH}$, Rodrigues EM. [Land transport injuries among emergency department visits in the state of Sao Paulo, in 2005]. Rev Saúde Pública. 2009;43(2):275-82. DOI: 10.1590/S0034-89102009000200008

23. Leguizamón R, Vega Bogado M. Epidemiología de los Accidentes de Tránsito en el Hospital Nacional. Itauguá Paraguay. Rev Nac(Itauguá). 2010;2(2):02-13.

24. Pavanitto DR, Menezes RAM, Nascimento LFC. Accidents involving motorcycles and potential years of life lost. An ecological and exploratory study. Sao Paulo Med J. 2018;136(1):4-9. DOI: 10.1590/15163180.2017.0098070817

25. Sutlovic D, Scepanovic A, Bosnjak M, Versic-Bratincevic M, Definis-Gojanovic M. The role of alcohol in road traffic accidents with fatal outcome: 10-year period in Croatia Split-Dalmatia County. Traffic Inj Prev. 2014;15(3):2227. DOI: $10.1080 / 15389588.2013 .804915$

26. García R, Abreu L. Seguridad vial en carreteras rurales de dos carriles. Rev Ing Construcción. 2016;31(1):54-60. DOI: $10.4067 / S 0718-50732016000100005$

27. Agudelo-Suarez AA, Duque-Serna FL, Restrepo-Molina L, Martinez-Herrera E. [Epidemiology of maxillofacial fractures due to traffic accidents in Medellin (Colombia)]. Gac Sanit. 2015;29 Suppl 1:30-5. DOI: 10.1016/j.gaceta.2015.04.007 